\title{
A Comparison between Two Vitrification Devices: Plastic Blade and McGill Cryoleaf
}

Maria Panagopoulou1, Nikos Nikolettos ${ }^{2}$, Vaia Dala², Elias Tsakos ${ }^{3}$, Konstantinos Charalabopoulos ${ }^{1}$ and Byron Asimakopoulos ${ }^{1 *}$

${ }^{1}$ Laboratory of Physiology, School of Medicine, Democritus University of Thrace, 68100 Alexandroupolis, Greece

${ }^{2}$ Laboratory of Reproductive Physiology, School of Medicine, Democritus University of Thrace, 68100 Alexandroupolis, Greece

${ }^{3}$ Gynaecology and Fertility Center, Thessaloniki, Greece

\begin{abstract}
Vitrification devices are of considerable importance for the successful vitrification of gametes and embryos They have to be easy to use and achieve good results. We compared the plastic blade, a new in-house device, to McGill Cryoleaf regarding the convenience of the use and the survival rate of vitrified human abnormal oocytes and embryos after thawing.
\end{abstract}

Material and methods: Abnormal oocytes and embryos derived from women who underwent I.V.F treatment were used in this study. Two groups were included. In the control group, abnormal oocytes and embryos were vitrified with the Cryoleaf; in the second group, it was used the plastic blade. In both groups, vitrification followed a standard and commercially available protocol.

Results: A number of problems appeared in the use of plastic blade during handling under liquid nitrogen and during placing the device into the warming solution. The survival rates of the vitrified oocytes $(61.53 \%)$ and embryos $(42.86 \%)$ with the plastic blade were significantly lower than with McGill Cryoleaf ( $80 \%$ and $61.54 \%$ respectively).

Conclusion: Plastic blade is not as effective as McGill Cryoleaf. Further improvements could establish it as an alternative vitrification device.

Keywords: Vitrification; Vitrification devices; Plastic blade; Cryoleaf

\section{Introduction}

The introduction of vitrification in the every-day clinical practice stimulated research on the vitrification devices. Today, several vitrification devices are available in the market with good clinical outcomes. Recently, Sugiyama et al. [1] introduced a novel and simple device made by a thin plastic board attached to a serum tube. This device, called "plastic blade", can be made in-house, it is very cheap and provides enough space to write on it information about the embryos. Sugiyama et al. [1] reported excellent results with this device, in terms of survival, implantation and pregnancy rates. The simplicity and the low cost of the plastic blade prompted us to test this device before its introduction in the clinical practice of our Department.

\section{Materials and Methods}

In this study, we compared plastic blade with McGill Cryoleaf (Origio, Denmark), that is a well- established vitrification device, regarding the convenience of the use and the survival rate of the vitrified oocytes and embryos after thawing. Plastic blade has not yet approved by the regularity authorities; therefore the study was conducted with abnormal oocytes and embryos coming from In-Vitro Fertilization (IVF) cycles carried on the IVF Unit of the General University Hospital of Alexandroupolis. The Scientific Committee of the General University Hospital of Alexandroupolis approved the research protocol and the patients signed an informed consent. The material came from patients followed ovarian stimulation for sperm injection and embryo transfer in the IVF Unit of the General University Hospital of Alexandroupolis from January to June 2011. The IVF procedures used in this study have been described elsewhere [2]. A total of 41 abnormal oocytes (at the germinal vesicle stage or at metaphases I, or metaphase II with a large polar body or abnormal shape) and 20 day 5 abnormal embryos (presenting a very low cleavage rate, cleavage arrest or a high degree of fragmentation) were included in the study. Fifteen oocytes and thirteen embryos were vitrified with McGill Cryoleaf whereas 26 oocytes and seven embryos were vitrified with the plastic blade. The oocytes and embryos were vitrified in single with both devices. The plastic blade was made according to the instructions of Sugiyama et al. [1]. A cryovial was used as vessel and polyethylene cut into a T-shaped piece was secured to the inner wall of the cup with the long arm standing out for loading the oocytes and embryos. The vitrification procedure with both devices was performed with Vitrification Cooling kit (Origio, Denmark). The vitrified material (oocytes or embryos) were stored in liquid nitrogen for two days. The thawing procedure was carried out with the Medicult Thawing kit (Origio, Denmark). The thawed oocytes and embryos were incubated in Universal IVF (Origio, Denmark) for two hours $\left(5 \% \mathrm{CO}_{2}\right.$, humified atmosphere, $37^{\circ} \mathrm{C}$ ). Then, the oocytes and embryos were carefully inspected under an inverted microscope. Statistical analysis of the results was performed with the Statistical Package for the Social Sciences 13.0 (SPSS Inc, Chicago, IL, USA). Comparisons between the two groups were made with the chi-square test. Differences were considered significant when $\mathrm{p}<0.05$.

\section{Results/Discussion}

The results obtained per experimental group are depicted in

*Corresponding author: Byron Asimakopoulos, Ph.D, Laboratory of Physiology School of Medicine, Democritus University of Thrace, 68100 Alexandroupolis, Greece, Tel: 30-2551030538; Fax: 30-2551030504; E-mail: basima@med.duth.gr

Received February 20, 2013; Accepted March 16, 2013; Published March 18, 2013

Citation: Panagopoulou M, Nikolettos N, Dala V, Tsakos E, Charalabopoulos K et al. (2013) A Comparison between Two Vitrification Devices: Plastic Blade and McGill Cryoleaf. Human Genet Embryol 3: 102. doi:10.4172/2161-0436.1000102

Copyright: (C) 2013 Panagopoulou M, et al. This is an open-access article distributed under the terms of the Creative Commons Attribution License, which permits unrestricted use, distribution, and reproduction in any medium, provided the original author and source are credited. 


\begin{tabular}{|l|c|c|c|c|}
\hline & McGill Cryoleaf & & \multicolumn{2}{|c|}{ Plastic blade } \\
\hline & Vitrified & Survived (\%) & Vitrified & Survived (\%) \\
\hline Oocytes & 15 & $12(80)^{\mathrm{a}}$ & 26 & $16(61.53)^{\mathrm{a}}$ \\
\hline Embryos & 13 & $8(61.54)^{\mathrm{b}}$ & 7 & $3(42.86)^{\mathrm{b}}$ \\
\hline Total & 28 & $21(75)^{\mathrm{c}}$ & 33 & $19(57.6)^{\mathrm{c}}$ \\
\hline
\end{tabular}

Values with the same superscript letters are significantly different $(p<0.05)$

Table 1: Survival rate of abnormal human oocytes and embryos vitrified with the plastic blade or McGill Cryoleaf.

table 1. Embryos as well as oocytes in the group of plastic blade had significantly lower survival rates than in the group of McGill Cryoleaf. The lower survival rate with the plastic blade is possible to be related with the thickness of polyethylene used in the plastic blade. The T-shaped polyethylene of the plastic blade is used for the loading of embryos and oocytes; this was made from thick polyethylene in order to be well attached into the cryovial. The thick polyethylene probably resulted in a slightly slower rate of warming. The slower warming rate could affect the success of the thawing procedure resulting in the lower survival rate recorded with the plastic blade. It is possible that the use of thinner polyethylene could overcome this problem.

The use of McGill Cryoleaf was free of problems during vitrification and thawing. However, a number of problems appeared with the use of the plastic blade. In eight cases, during the immersion into the liquid nitrogen, the plastic blade was detached from the McGill Cryoleaf.
There were also difficulties in handling the plastic blade into the liquid nitrogen which exposed hands in very low temperatures. This problem had to do with the short length of the plastic blade. The use of long forceps did not solve the problem sufficiently. Long devices, as McGill Cryoleaf, offer an easier and safer handling into the liquid nitrogen.

The cost of vitrification devices is an important parameter for IVF centers. In this context, the plastic blade has a clear advantage as it is made in-house with a cost around 200 times lower than the price of McGill Cryoleaf.

In conclusion, the results of the present study showed that the plastic blade described by Sugiyama et al. [1] is a cheap and effective open device for vitrification, though not so effective as McGill Cryoleaf. To our opinion, the plastic blade is susceptible of further improvements in order to become a good alternative vitrification device.

\section{References}

1. Sugiyama R, Nakagawa K, Shirai A, Sugiyama R, Nishi Y, et al. (2010) Clinical outcomes resulting from the transfer of vitrified human embryos using a new device for cryopreservation (plastic blade). J Assist Reprod Gen 27: 161-167.

2. Nikolettos N, Asimakopoulos B, Diedrich K, Al-Hasani S (2004) Triptorelin versus cetrorelix in intracytoplasmic sperm injection cycles in women with a single ovary. Eur J Obstet Gynecol Reprod Biol 112: 185-188. 